AperTO - Archivio Istituzionale Open Access dell'Università di Torino

\title{
Assessing personality using emoji: An exploratory study
}

\section{This is the author's manuscript}

Original Citation:

Availability:

This version is available http://hdl.handle.net/2318/1640253

since 2017-05-29T12:03:21Z

Published version:

DOI:10.1016/j.paid.2017.02.037

Terms of use:

Open Access

Anyone can freely access the full text of works made available as "Open Access". Works made available under a Creative Commons license can be used according to the terms and conditions of said license. Use of all other works requires consent of the right holder (author or publisher) if not exempted from copyright protection by the applicable law. 
Title: "Assessing personality using emoji: an exploratory study."

Running Title: “Assessing personality using emoji."

Davide Marengo $^{\mathrm{a}}$, Fabrizia Giannotta ${ }^{\mathrm{b}}$, Michele Settanni ${ }^{\mathrm{a} *}$

${ }^{a}$ Department of Psychology, University of Turin, Italy.

${ }^{\mathrm{b}}$ Uppsala University, Uppsala Child and Baby Lab, Department of Psychology, Uppsala, Sweden

*Corresponding author

The corresponding author can be reached at the following address:

Michele Settanni

Department of Psychology, University of Turin, Italy.

Via Verdi, 1010124 Torino, Italy

Phone: 00390116702793

michele.settanni@unito.it 


\section{Highlights}

- Emoji are pictorial symbols widely used in computer-mediated communication

- We studied the relationship between self-identification with emoji and personality traits

- Significant correlations emerged with neuroticism, extraversion and agreeableness

- Emoji-based personality scores show adequate psychometric characteristics 
*Manuscript without author identities

Click here to view linked References

Title: "Assessing personality using emoji: an exploratory study."

Running Title: “Assessing personality using emoji." 


\begin{abstract}
The increasing popularity of text-based computer mediated communication, such as instant messaging and mobile texting, have resulted in the emergence of a new pictographic form of language, i.e. emoji, offering an intuitive and informal way to convey emotions and attitudes, replacing words or phrases in text messages. Based on these characteristics, could identification with emoji be associated with personality? Could they be used instead of text-based items in personality assessment? The present study aimed at exploring these questions. The sample is composed of 234 young adults recruited online (age: $\mathrm{M}=24.79, \mathrm{SD}=6.47 ; 62 \%$ female). Participants responded to a brief Big-five personality questionnaire and a 91-item survey assessing participants' degree of self-identification with emoji selected from the Apple Color Emoji fontset. Results indicated that 36 out of 91 examined emoji are significantly related with three of the Big-five personality traits - emotional stability, extraversion, and agreeableness - that are consistently linked with emotion and affective processing. Emoji-based measures of these personality traits show moderate-to-large concurrent validity with scores from a validated personality questionnaire $(\mathrm{r}=0.6-0.8)$. Overall, our study advances the idea that emoji might be employed to develop a language-free assessment tool for personality.
\end{abstract}




\section{Introduction}

\subsection{Non-verbal elements in Computer Mediated Communication (CMC)}

The relatively recent growth in popularity of text-based CMC - e.g., email, instant messaging, and mobile texting - has been accompanied by the birth of new forms of language. Among these, one of the newest and most distinctive are emoticons (punctuation-based renditions of facial expressions, objects and symbols, e.g., :-) or :-P) and their update in pictographic form, emoji (e.g., the Apple Color Emoji fontset, see Table 1 for examples). Emoji, in particular, cover a broader and ever-increasing range of areas and subjects and are used by 92 percent of the online population, with females and young people under 30 being the most frequent users (EMOGI, 2015). They are increasingly employed, especially among young people, as a rapid, informal way to convey emotions (Walther \& D'Addario, 2001) and attitudes (e.g., sarcasm, Dresner \& Herring, 2010) in instant messaging services (e.g., Apple iMessage or Whatsapp Messenger, Statista, 2013), and in social media (Dimson, 2015). From a linguistic point of view, emoticon and emoji appear to work as actual words (Pierozak, 2003), to serve as a surrogate of non-verbal cues and to contribute to the overall meaning of written CMC messages (Walther \& D'Addario, 2001; Derks, Fisher \& Bos, 2008; Jibril \& Abdullah, 2013). Recent studies indicate that individual differences in emoticon and emoji use in CMC tend to echo differences in psychological characteristics. For example, Hall and Pennington (2013) found frequency of emoticon use among Facebook users to be positively associated with extraversion and selfmonitoring traits. Similarly, Settanni and Marengo (2015) found use of emoticons expressing positive sentiment in Facebook posts to be negatively associated with users' emotional distress. Given these characteristics, it can be hypothesized that emoji could be used in psychological assessments as a language-free alternative to items that usually require a certain level of literacy and fluency in a specific language. To our knowledge, only one study addressed this issue by 
exploring the use of emoji-based instruments for the detection of depressive symptoms in stroke patients (Lee, Tang, Yu \& Cheung, 2008). Their findings demonstrate that the emoji-based items can provide a measure of depression as reliable as traditional text-based items. This calls for a further and deeper exploration of the use of emoji as assessment tools.

\subsection{Verbal stimuli in personality assessment}

Personality differences are widely and commonly assessed through lexical stimuli, e.g. personality-descriptive adjectives or phrases. Well-known examples of this method are tests based on the Five Factor personality trait model (McCrae \& Costa, 2008), such as the NEO-PI (Costa \& McCrae, 1992), the Big Five Inventory (John, Donahue, \& Kentle, 1991), the Big Five Questionnaire (Caprara, Barbaranelli, Borgogni, \& Perugini, 1993), and the TIPI questionnaire (Gosling, Rentfrow \& Swann, 2003). The idea behind this approach is known as the "lexical hypothesis", which posits that words and expressions commonly used by people tend to reflect individual differences (Goldberg, 1981, see also De Raad, Perugini, Hrebickova, \& Szarota, 1998; Goldberg, 1982, 1993; Saucier \& Goldberg, 1996, 2001). However, a recognized limitation linked to these tests is the measurement bias due to respondents' language and educational level (Rolland, Parker \& Stumpf, 1998; John \& Srivastava, 1999; Rammstedt, Goldberg \& Borg, 2010). A possible countermeasure against these sources of bias might consist of the adoption of language-independent items. In support of that, previous studies demonstrate that picture-based items have been successfully employed in other areas of psychological measurement, such as intelligence (Naglieri, 2003) and neuropsychological assessment (for a review, see McCallum, 2003).

Given the widespread use of emoji and their functional similarity with words, could emoji be used instead of verbal items to assess individual personality? To our knowledge, no studies have attempted to answer this question. This study aims to fill this gap within the literature. 


\subsection{Aims}

The present study aims to explore whether emoji-based items can be used to assess personality traits. We pursued our aim by administering a questionnaire composed of a brief validated personality measure and a large set of items assessing participants' degree of self-identification with emoji extracted from the popular Apple Color Emoji fontset.

\section{Material and methods}

\subsection{Participants and procedure}

Participants were recruited online through snowball sampling. Participants were 234 young English-speaking adults (mean age: $24.79, \mathrm{SD}=6.47 ; 62 \%$ female). Participants did not receive any compensation for their participation and completion of the study. The anonymous survey was hosted on the site Survey monkey (www.Surveymonkey.com), a secure website used to collect survey-based data for research. Initially, 5 university students were enrolled in the study and asked to advertise the research amongst two social-media platforms (Reddit and Facebook) by publishing the link to the survey webpage. The survey took place in March 2016 and two hundred forty-one users (63\% Reddit users; 37\% Facebook users) checked the research page, 234 of which completed the survey.

\subsection{Emoji survey}

The emoji used in the present study were extracted from one the earliest emoji fontset, the Apple Color Emoji fontset, which includes more than 1600 emoji (for a complete list, see http://emojipedia.org/apple/). The Apple Color Emoji fontset is available in the popular Apple's iMessage and Facebook's Whatsapp instant messaging services, which serve over one billion users combined. Given the high number of emoji included in the Apple Color fontset, a 
preliminary selection of emoji was performed through three focus groups each involving 8 master degree students enrolled in psychology classes at the University of Turin, Italy. Students were asked to (1) discuss the relationship between emoji and personality features and (2) select a list of emoji that, in their opinion, would be able to represent their and their friends' personality characteristics. Eventually, 91 emoji appearing in at least two of the three obtained lists were selected for inclusion in the online survey. The included emoji set mainly consisted of those representing faces or people (68 out of 91). The others were mainly emoji depicting objects (e.g., bomb), symbols (e.g., stylized heart) and other anthropomorphic emoji (e.g., hand-gestures). A questionnaire investigating self-identification with emoji was then constructed using the selected emoji as stimuli. The questionnaire consisted of 91 items, each depicting an emoji, with the following common stem: "How do you recognize yourself in the following emoji?". Items were scored using a 5-point Likert scale ranging from 1 ("Not like me at all") to 5 ("Very much like me"). In order to mitigate potential bias related to presentation order effects, item order was randomized at each online survey administration.

\subsection{Personality traits}

Personality differences were assessed by administering the Ten-Item Personality Inventory (TIPI) (Gosling Rentfrow \& Swann, 2003), a short measure assessing the Big-Five personality traits of extroversion, agreeableness, conscientiousness, emotional stability, and openness to new experiences. The instrument consists of 10 items ( 2 items per trait) with a common stem of 'I see myself as'. Each item is rated on a 7-point scale ranging from 1 (disagree strongly) to 7 (agree strongly). In spite of its brevity, the TIPI personality scales have shown good psychometric properties when compared to longer instruments assessing Big-Five personality traits (Gosling, Rentfrow \& Swann, 2003). As expected, given the low number of items per trait, internal consistency for this instrument was not high, ranging from $\alpha=.37$ for Agreeableness to $\alpha=.66$ 
for Extraversion (mean $\alpha=.52$ ). However, these values are in line with the ones presented in TIPI validation studies (e.g. Gosling, 2003; Romero, Villar, Gómez-Fraguela, \& López-Romero, 2012).

\subsection{Analytic strategy}

Participants' degree of self-identification with emoji was investigated by computing a set of descriptive statistics on participants' ratings to the emoji stimuli. Specifically, we looked at: 1. the percentage of participants who reported a partial-to-total self-identification with at least one of the presented emoji (response score $>1$ ); 2 . the average number of emoji participants selfidentified with (response score $>1$ ); 3 . the percentage of participants who reported the highest level of identification with at least one emoji (response score $=5$ ). We also computed the mean score and standard deviation for each emoji.

In order to study the relationship between participants' self-identification with emoji and personality traits, for each emoji we computed the correlations between participants' selfidentification ratings and the TIPI personality trait scores. Due to the high number computed correlations (91 emoji per 5 personality traits), Bonferroni correction was applied to the nominal alpha level of .05 , yielding a corrected alpha level of .0001 .

In order to further investigate the possibility of creating a brief emoji-based instrument to assess personality, we conducted an exploratory factor analysis (EFA) on the emoji items showing the highest correlations with each TIPI trait.

Then, based on the EFA results, a confirmatory factor analysis (CFA) was performed to examine whether the Big Five theoretical model fit the data. Based on CFA results, emoji-based personality scores were computed as unweighted sums of items comprising each trait. As a last step, concurrent validity was examined by computing the correlations between TIPI personality scores and emoji-based scores of the same traits. In order to rule out potential age or gender- 
related bias in emoji-based measurement, we used Z-tests to test for age- and gender-differences in correlations between TIPI and emoji-based trait scores.

\section{Results}

\subsection{Item functioning}

The large majority of participants $(96.2 \%)$ provided a partial-to-total self-identification rating with at least one of the 91 presented emoji (response rating $>1$ ), while $69.7 \%$ of participants reported the highest level of identification (response rating $=5$ ) with at least one emoji stimuli. The average number of emoji participants self-identified with (response rating $>1$ ) was 53.24 $(\mathrm{SD}=23.59)$. The emoji reporting the highest self-identification level was $\odot(\mathrm{M}=3.16$, $\mathrm{SD}=1.42$ ). The emoji associated with both the lowest level of identification and score variability was $(\mathrm{M}=1.40, \mathrm{SD}=0.87)$ while the emoji reporting the higher variability in scores was $(\mathrm{M}=1.77, \mathrm{SD}=1.50)$

\subsection{Item correlations with personality traits}

Table 1 reports the 36 (out of 91) emoji for which participants' self-identification ratings showed a significant correlation with at least one of the TIPI personality traits. The remaining 55 emoji did not significantly correlate with TIPI traits. Only three personality traits were found to correlate with emoji ratings: extraversion, emotional stability and agreeableness. No significant correlations emerged with conscientiousness and openness to experiences. When examining the correlations, a specific pattern seemed to emerge. 
Table 1.

Correlations between emoji identification scores and TIPI personality traits

\begin{tabular}{|c|c|c|c|c|c|c|c|}
\hline Emoji & Unicode name & Mean (SD) & Agree. & Extrav. & Em.St. & Open. & Cons. \\
\hline (1) & Smiling face with smiling eyes & $3.14(1.40)$ & 0.37 & 0.18 & 0.00 & 0.07 & 0.17 \\
\hline$\Leftrightarrow$ & White smiling face & $2.94(1.40)$ & 0.33 & 0.21 & 0.00 & 0.09 & 0.13 \\
\hline$=$ & Kissing face with closed eyes & $2.37(1.33)$ & 0.29 & 0.23 & -0.11 & 0.09 & 0.09 \\
\hline$\theta$ & Smiling face with sunglasses & $2.76(1.39)$ & 0.00 & 0.41 & 0.07 & 0.11 & 0.07 \\
\hline$\Leftrightarrow$ & Winking face & $2.87(1.35)$ & 0.13 & 0.37 & 0.17 & 0.07 & 0.10 \\
\hline$\theta$ & Smiling face with open mouth & $2.95(1.36)$ & 0.12 & 0.36 & 0.19 & 0.00 & 0.15 \\
\hline$\hat{\theta}$ & Smiling face with open mouth and smiling eyes & $2.89(1.28)$ & 0.18 & 0.34 & 0.17 & 0.02 & 0.11 \\
\hline$\Leftrightarrow$ & Face with stuck-out tongue and winking eye & $2.55(1.42)$ & 0.05 & 0.32 & 0.13 & -0.02 & 0.09 \\
\hline 6 & Flexed biceps & $2.03(1.31)$ & 0.07 & 0.32 & 0.10 & 0.10 & 0.05 \\
\hline \%) & Face throwing a kiss & $2.88(1.48)$ & 0.23 & 0.30 & -0.05 & 0.13 & 0.13 \\
\hline$\Leftrightarrow$ & Face with tears of joy & $3.14(1.42)$ & 0.03 & 0.29 & -0.01 & 0.06 & 0.04 \\
\hline$\Theta$ & Face savouring delicious food & $2.44(1.31)$ & 0.10 & 0.28 & 0.09 & 0.00 & 0.19 \\
\hline (20) & Smiling face with heart-shaped eyes & $2.91(1.46)$ & 0.20 & 0.28 & -0.1 & 0.08 & 0.11 \\
\hline & Party popper & $2.39(1.37)$ & 0.09 & 0.28 & 0.13 & 0.01 & 0.14 \\
\hline & Heavy black heart & $3.15(1.41)$ & 0.23 & 0.28 & -0.05 & 0.16 & 0.18 \\
\hline 8 & Victory hand & $2.38(1.36)$ & 0.17 & 0.28 & 0.08 & 0.04 & 0.04 \\
\hline 4 & Fisted hand sign & $1.96(1.22)$ & -0.11 & 0.27 & 0.09 & 0.03 & 0.12 \\
\hline 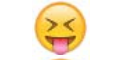 & Face with stuck-out tongue and tightly-closed eyes & $2.33(1.32)$ & 0.03 & 0.26 & 0.01 & 0.05 & 0.09 \\
\hline$=$ & Pensive face & $2.51(1.25)$ & 0.07 & -0.04 & -0.43 & -0.07 & -0.09 \\
\hline$\Leftrightarrow$ & Disappointed face & $2.36(1.19)$ & 0.10 & -0.09 & -0.39 & -0.08 & -0.10 \\
\hline$\because \because$ & Disappointed but relieved face & $2.31(1.23)$ & 0.07 & -0.06 & -0.36 & 0.00 & 0.04 \\
\hline & Face with open mouth and cold sweat & $2.09(1.21)$ & 0.00 & -0.09 & -0.35 & -0.01 & -0.03 \\
\hline$\approx$ & Face with cold sweat & $2.18(1.17)$ & 0.03 & -0.12 & -0.34 & -0.06 & -0.07 \\
\hline $60^{\circ}$ & Crying face & $2.23(1.12)$ & 0.09 & -0.03 & -0.34 & -0.06 & -0.05 \\
\hline sicis & Confounded face & $1.85(1.15)$ & 0.07 & 0.00 & -0.33 & 0.03 & -0.12 \\
\hline (3) & Persevering face & $2.03(1.08)$ & 0.09 & -0.13 & -0.32 & -0.10 & -0.04 \\
\hline 3 & Tired face & $2.09(1.21)$ & 0.11 & -0.05 & -0.30 & -0.02 & 0.02 \\
\hline$=$ & Sleepy face & $1.97(1.12)$ & 0.05 & -0.03 & -0.30 & -0.04 & -0.02 \\
\hline 2 & Weary face & $2.23(1.22)$ & 0.10 & 0.03 & -0.28 & -0.07 & 0.03 \\
\hline$\therefore$ & Fearful face & $2.11(1.18)$ & 0.05 & 0.04 & -0.28 & -0.01 & -0.06 \\
\hline$\because$ & Anguished face & $2.15(1.07)$ & 0.00 & -0.06 & -0.28 & -0.1 & -0.04 \\
\hline $2=$ & Face with look of triumph & $1.95(1.18)$ & -0.14 & 0.06 & -0.27 & 0.06 & 0.04 \\
\hline$\because$ & Worried face & $2.07(1.07)$ & -0.03 & -0.03 & -0.27 & -0.06 & -0.02 \\
\hline$\therefore$ & Face with head-bandage & $1.64(0.95)$ & -0.07 & -0.04 & -0.26 & -0.18 & 0.02 \\
\hline 608 & Face screaming in fear & $2.41(1.26)$ & 0.04 & 0.16 & -0.26 & 0.07 & -0.05 \\
\hline$\frac{x}{e}$ & Astonished face & $1.67(1.02)$ & -0.01 & 0.01 & -0.25 & -0.03 & -0.14 \\
\hline
\end{tabular}

Note: $\mathrm{N}=234$. In bold: Correlations significant at Bonferroni-corrected $\mathrm{p}<.05$. Agree.: Agreeableness; Extrav.:

Extraversion; Em.St.: Emotional Stability; Open.: Openness to New Experiences; Cons.: Conscientiousness. 
Self-identification with emoji conveying negative affect (e.g., anger, sadness, disappointment, weariness) negatively correlated (range $r=-.43$ to -.25 ) with the emotional stability trait score. Conversely, self-identification with emoji depicting faces, objects and hand- and body- gestures expressing positive emotions (e.g., happiness, love, parties) showed significant positive correlations (range $r=.26$ to .41 ) with the extraversion trait score. One exception to our findings is found in emoji depicting blushing faces expressing positive emotions (i.e., smiling or kissing) which positively correlated (range $r=.29$ to .37 ) with agreeableness.

\subsection{Factor structure and correlations with TIPI traits}

Given the absence of emoji items significantly correlated with openness and conscientiousness as measured by TIPI, these two traits were not considered in subsequent analyses. In order to explore the factor structure of the emoji-based instrument, an EFA was conducted on 9 items: in the analysis we included the three emoji-based items that showed the strongest correlation with the emotional stability, agreeableness and extraversion TIPI scores. We excluded items that significantly correlated with more than one TIPI trait. EFA was carried out using weighted least square mean and variance adjusted estimation (WLSMV) with Geomin rotation. Fit between the model and the data was evaluated using $\chi^{2}$, comparative fit index (CFI), root mean square error of approximation (RMSEA), and standardized root mean square residual (SRMR). CFI $\geqslant 0.90$, RMSEA $<0.05$, and SRMR $<0.05$ indicate a good fit to the data (Browne \& Cudeck, 1993, Cooke et al., 2013). The number of factors to retain was chosen based on a combination of methods including the Scree plot examination and by considering the difference in fit between nested models. A three-factor solution emerged as most appropriate $\left(\chi^{2}=19.73 ; \mathrm{df}=12 ; \mathrm{CFI}=\right.$ .99 ; RMSEA $=.05 ; \mathrm{SRMR}=.02$ ), explaining $59.7 \%$ of variance. A CFA was then conducted to test the fit of the selected emoji items to the three-dimensional model expected on the basis of 
theoretical considerations, with the 9 items loading on the three factors. Model fit was good $\left(\chi^{2}=\right.$ 41.09; $\mathrm{df}=24 ; \mathrm{CFI}=.99 ; \mathrm{RMSEA}=.05)$. Given these promising results, we further tested concurrent validity by computing the correlation between TIPI subscales and emoji-based summed scores. Due to low internal consistency of the TIPI measures, correlations were corrected for attenuation due to measurement error, as suggested by Gosling and colleagues (2003) and done by many authors (e.g., Hahn Gottschling \& Spinath, 2012; Jonason \& Webster, 2010; Heller, Komar \& Lee, 2007). Internal consistency and correlation with corresponding traits are reported in Table 2. Reliability of emoji-based measures was good $(\alpha \geq .72)$. Correlations between TIPI dimensions and emoji-based measures were large, ranging from .68 to .83 with a mean correlation of .73. As a final step, correlations were examined for differences by gender and age group (Young adults: Age $\leq 25$; Old adults: Age $\geq 26$ ). Results of Z-tests showed no significant differences in correlations between TIPI and emoji-based scores by age (Agreeableness: $\mathrm{Z}=1.54, \mathrm{p}=.13$; Extraversion: $\mathrm{Z}=-0.09, \mathrm{p}=.93$; Emotional stability: $\mathrm{Z}=$ $1.33, \mathrm{p}=.18$ ) and gender (Agreeableness: $\mathrm{Z}=-1.10, \mathrm{p}=.27$; Extraversion: $\mathrm{Z}=1.13, \mathrm{p}=.26$; Emotional stability: $\mathrm{Z}=0.48, \mathrm{p}=.63$ ).

\section{Table 2.}

Emoji-based personality scores: Selected emoji items, reliability and correlation with TIPI dimensions.

\begin{tabular}{lrrc}
\hline Trait & Selected emoji & $\boldsymbol{\alpha}$ & $\begin{array}{c}\text { Correlation } \\
\text { with TIPI dimensions }\end{array}$ \\
\hline Agreeableness & & .81 & .83 \\
Extraversion & & .72 & .68 \\
Emotional stability & & .77 & .68 \\
\hline
\end{tabular}

Note: Bolded squares indicate reversed items 


\section{Discussion}

The present study aimed at investigating whether self-identification with various emoji could provide information related to personality differences. In order to achieve this aim we examined the functioning of a large set of emoji-based items and we evaluated the associations between participants' responses and personality traits.

Overall, the examination of answers to emoji items showed that participants used the full range of responses and that emoji items had generally high standard deviations, hence evidencing an adequate response variability. In regards to the relationship with personality traits, 36 out of the 91 administered emoji showed significant associations with three of the five TIPI personality traits. More in detail, the emoji were significantly related with the traits that have shown the most consistent links with emotions and affective processing - i.e., emotional stability, extraversion and agreeableness (Côté \& Moskowitz, 1998; Elliot \& Thrash, 2002; Robinson, 2007). In turn, we found no associations between emoji and both the conscientiousness and openness to experience traits. This might be explained with the fact that these traits are not typically linked with emotional expression, but instead they show strong links with general cognitive ability (LePine, Colquitt \& Erez, 2000; Moutafi, Furnham \& Crump, 2006).

Overall, the direction of the associations is consistent with findings from literature investigating associations between personality traits and affect (Côté \& Moskowitz, 1998). More in details, neuroticism (emotional stability viewed from the negative pole) showed strong relationship with emoji conveying negative affect, while extraversion revealed consistent associations with items depicting emoji conveying positive emotions. Indeed, these results are in accordance with previous research, which found that individuals with high levels of neuroticism tend to experience higher levels of worry and negative affect than individuals low on this trait (Elliot \& Thrash, 2002; Watson \& Clark, 1984). In addition, extraverts have been shown to experience 
higher levels of positive affect than introverts (Côté \& Moskowitz, 1998; Lucas \& Diener, 2001). Lastly, we found an interesting pattern concerning emoji depicting blushing faces, which only correlated with the agreeableness trait. The specific association between the blushing emoji and the agreeableness trait may be interpreted in light of the literature indicating blushing as a signal promoting positive social interactions. Indeed, blushing has been shown to convey sincerity (Dijk, de Jong \& Peters, 2009), flirtation (Elliot \& Niesta, 2008), and to mitigate negative social impression (Keltner, Young \& Buswell, 1997). Hence, self-identification with emoji depicting both positive emotions and blushing might reflect individuals' tendency to see and present themselves as benevolent, other-focused and straightforward, which in turn are distinctive characteristics of individuals scoring high on the agreeableness trait. However, this hypothesis should be further explored.

Finally, emoji-based measures of personality traits show quite high correlation with TIPI dimensions, indicating good concurrent validity between the measures. This result is quite impressive and suggests that the new forms of communication, such as emoji should be explored further as they have great potential to replace, at least in part, the traditional instruments to assess individual personality differences. Taking into account the popularity of this type of new communication, the immediacy of the messages they convey, and the fact that they are languagefree, leads us to believe that emoji might represent a new way to assess personality differences across populations different for language and literacy level. This study is a first step toward that direction.

Our study has some weaknesses. The main limitation is that the adopted online snowball sampling, might have introduced a potential self-selection bias, which may negatively affect the representativeness of the population. However, as illustrated by Bauermeister et al., (2012), studies conducted using this kind of sampling are especially suitable for young adults and, as 
noted by Kosinski et al., (2015), they are not necessarily affected by stronger biases than the ones employing other recruitment approaches. Moreover, the relatively small sample did not allow testing for cross invariance among boys and girls. Future replications employing larger, more controlled samples could help strengthen the result of the study.

This study however also contains strengths. First, we were able to evaluate if self-identification with emoji is relevantly linked with personality. Second, the use of very strict of level of significance in correlation analyses make our results robust and trustful.

Third and foremost, to our knowledge this is the first study to investigate the possibility to use a widespread language-free form of expression - i.e., emoji - to study personality. In doing so, our study suggests that emoji might be useful in psychological assessment for the study of personality traits, especially those with known connections to emotional expression and affect. This finding opens up new frontiers in the development of tools to study personality differences in diverse populations. 


\section{References}

Bauermeister, J. A., Zimmerman, M. A., Johns, M. M., Glowacki, P., Stoddard, S., \& Volz, E. (2012). Innovative recruitment using online networks: lessons learned from an online study of alcohol and other drug use utilizing a web-based, respondent-driven sampling (webRDS) strategy. Journal of Studies on Alcohol and Drugs, 73(5), 834-838.

Costa, P. T., \& McCrae, R. R. (1992). NEO PI-R: NEO Personality Inventory-Revised. Psychological Assessment Resources.

Caprara, G. V., Barbaranelli, C., Borgogni, L., \& Perugini, M. (1993). The "Big Five Questionnaire": A new questionnaire to assess the five factor model. Personality and individual Differences, 15(3), 281-288.

Côté, S., \& Moskowitz, D.S. (1998). On the dynamic covariation between interpersonal behavior and affect: prediction from neuroticism, extraversion, and agreeableness. Journal of personality and social psychology, 75(4), 1032-1046.

Derks, D., Fischer, A.H., \& Bos, A.E. (2008). The Role of Emotion in Computer-Mediated Communication: A Review. Computers in Human Behavior, 24, 766-785.

Dijk, C., de Jong, P.J., \& Peters, M.L. (2009). The remedial value of blushing in the context of transgressions and mishaps. Emotion, 9(2), 287-291.

Dimson, T., Emojineering part 1: Machine learning for emoji trends [blog]; 2015. Retrieved from http://instagram-engineering.tumblr.com/post/117889701472/emojineering-part-1machine-learning-for-emoji/. 
Dresner, E. \& Herring, S.C. (2010). Functions of the nonverbal in CMC: Emoticons and illocutionary force. Communication theory, 20 (3), 249-268.

Elliot, A.J., \& Niesta, D. (2008). Romantic red: red enhances men's attraction to women. Journal of personality and social psychology, 95(5), 1150.

Elliot, A.J., \& Thrash, T.M. (2002). Approach-avoidance motivation in personality: approach and avoidance temperaments and goals. Journal of personality and social psychology, 82(5), 804-818.

EMOGI. (2015). Emoji report.

Retrieved from http://emogi.com/documents/Emoji_Report_2015.pdf

Gosling, S.D., Rentfrow, P.J., \& Swann, W.B. (2003). A very brief measure of the Big-Five personality domains. Journal of Research in personality, 37(6), 504-528.

Hall, J.A., \& Pennington, N. (2013). Self-monitoring, honesty, and cue use on Facebook: The relationship with user extraversion and conscientiousness. Computers in Human Behavior, 29(4), 1556-1564.

Hahn, E., Gottschling, J., \& Spinath, F. M. (2012). Short measurements of personality-Validity and reliability of the GSOEP Big Five Inventory (BFI-S).Journal of Research in Personality, 46(3), 355-359.

Heller, D., \& Komar, J., \& Lee, W. B. (2007). The dynamics of personality states, goals, and well-being. Personality and Social Psychology Bulletin, 33(6), 898-910.

Jonason, P. K., \& Webster, G. D. (2010). The dirty dozen: a concise measure of the dark triad. Psychological assessment, 22(2), 420. 
Jibril, T.A., \& Abdullah, M.H. (2013). Relevance of emoticons in computer-mediated communication contexts: An overview. Asian Social Science, 9(4), 201-207.

Keltner, D., Young, R.C., \& Buswell, B.N. (1997). Appeasement in human emotion, social practice, and personality. Aggressive Behavior, 23, 359-374.

Kosinski, M., Matz, S. C., Gosling, S. D., Popov, V., \& Stillwell, D. (2015). Facebook as a research tool for the social sciences: Opportunities, challenges, ethical considerations, and practical guidelines. American Psychologist, 70(6), 543.

Kwak, J. (2014). Why So Serious?: Detecting and Tracking Depression Through the Presence of Emoticons. In: Medicine 2.0 Conference. JMIR Publications Inc.

Lee, A. C. K., Tang, S.W., Yu, G. K. K., \& Cheung, R. T. F. (2008). The smiley as a simple screening tool for depression after stroke: A preliminary study. International journal of nursing studies, 45(7), 1081-1089.

LePine, J. A., Colquitt, J. A., \& Erez, A. (2000). Adaptability to changing task contexts: Effects of general cognitive ability, conscientiousness, and openness to experience. Personnel psychology, 53(3), 563-593.

Lucas, R. E. \& Diener, E. (2001). Understanding extraverts' enjoyment of social situations: the importance of pleasantness. Journal of personality and social psychology, 81(2), 343356.

McCallum, R. S. (2003). Handbook of nonverbal assessment. Springer Science \& Business Media. 
Moutafi, J., Furnham, A., \& Crump, J. (2006). What facets of openness and conscientiousness predict fluid intelligence score? Learning and Individual Differences, 16(1), 31-42.

Naglieri, J. A. (2003). Naglieri nonverbal ability tests. In Handbook of nonverbal assessment (pp. 175-189). Springer US.

Robinson, M. D. (2007). Personality, Affective Processing, and Self- Regulation: Toward Process- Based Views of Extraversion, Neuroticism, and Agreeableness. Social and Personality Psychology Compass, 1(1), 223-235.

Romero, E., Villar, P., Gómez-Fraguela, J. A., \& López-Romero, L. (2012). Measuring personality traits with ultra-short scales: A study of the Ten Item Personality Inventory (TIPI) in a Spanish sample. Personality and Individual Differences, 53(3), 289-293.

Settanni, M., \& Marengo, D. (2015). Sharing feelings online: studying emotional well-being via automated text analysis of Facebook posts. Frontiers in psychology, 6.

Statista. (2013). Sticker and emoji usage in mobile messaging apps 2013. Retrieved from http://www.statista.com/statistics/301061/mobile-messaging-apps-sticker-emoji-usage/

Walther, J.B. \& D'Addario K.P. (2001). The impacts of emoticons on message interpretation in computer-mediated communication. Social science computer review, 19(3), 324-347.

Watson, D., \& Clark, L.A. (1984). Negative affectivity: the disposition to experience aversive emotional states. Psychological bulletin, 96(3), 465-490. 